\title{
A Locust Phase Change Model with Multiple Switching States and Random Perturbation
}

\author{
Changcheng Xiang \\ Key Laboratory of Biologic Resources Protection and Utilization, \\ Hubei University for Nationalities, Enshi, \\ Hubei 445000, P. R. China \\ xcc7426681@126.com \\ Sanyi Tang* \\ School of Mathematics and Information Science, \\ Shaanxi Normal University, Xi'an 710062, P. R. China \\ sytang@snnu.edu.cn \\ Robert A. Cheke \\ Natural Resources Institute, University of Greenwich at Medway, \\ Central Avenue, Chatham Maritime, Chatham, \\ Kent ME4 4TB, UK \\ r.a.cheke@greenwich.ac.uk \\ Wenjie Qin \\ College of Science, China Three Gorges University, \\ Yichang 443002, P. R. China \\ wenjieqin@hotmail.com
}

\begin{abstract}
Insects such as locusts and some moths can transform from a solitarious phase when they remain in loose populations and a gregarious phase, when they may swarm. Therefore, the key to effective management of outbreaks of species such as the desert locust Schistocercagregaria is early detection of when they are in the threshold state between the two phases, followed by timely control of their hopper stages before they fledge because the control of flying adult swarms is costly and often ineffective. Definitions of gregarization thresholds should assist preventive control measures and avoid treatment of areas that might not lead to gregarization. In order to better understand the effects of the threshold density which represents the gregarization threshold on the outbreak of a locust population, we developed a model of a discrete switching system. The proposed model allows us to address: (1) How frequently switching occurs from solitarious to gregarious phases and vice versa; (2) When do stable switching transients occur, the existence of which indicate that solutions with larger amplitudes can switch to a stable attractor with a value less than the switching threshold density?; and (3) How does random perturbation influence the switching pattern? Our results show that both subsystems have refuge equilibrium points, outbreak equilibrium points and bistable equilibria. Further, the outbreak equilibrium points and bistable equilibria can coexist for a wide range of parameters and can switch from one to another. This type of switching is sensitive to the intrinsic growth rate and the initial values of the locust population, and may result in locust population outbreaks and phase switching once a small perturbation occurs. Moreover, the simulation results indicate that the switching transient patterns become identical after some generations, suggesting that the
\end{abstract}

\footnotetext{
*Author for correspondence
} 
evolving process of the perturbation system is not related to the initial value after some fixed number of generations for the same stochastic processes. However, the switching frequency and outbreak patterns can be significantly affected by the intensity of noise and the intrinsic growth rate of the locust population.

Keywords: Discrete switching system; stable switching transient; phase change; random perturbation; bistability.

\section{Introduction}

Insects such as locusts and some moths are polyphenic. They can change their "phase" from one condition to another, usually in response to changes in their population densities. Locusts are distinguished from grasshoppers by this ability. They spend much of their lives in a "solitarious" phase, behaving just like any other species of grasshopper, until an increase in their density will cause them to begin a switch to being in a "gregarious" phase [Uvarov, 1921; Uvarov et al., 1977]. For instance, the desert locust Schistocercagregaria changes color, body dimensions, physiology and behavior as it enters a "transiens" phase before becoming fully gregarious when, as this name implies, it becomes highly social. The developing stages, within hopper bands, alter from being greenish to black and yellow and moult to become swarming adults. These swarms move by day, in contrast to the nocturnal movements of solitarious desert locusts, and are capable of very long distance migrations following characteristic pathways determined by the season and prevailing meteorological conditions [Pedgley, 1981, 1980]. The mechanisms eliciting phase changes in locusts and noctuid moths are many and varied, including tactile responses with increasing densities [Simpson et al., 1999] which in turn are triggered by environmental influences [Cheke \& Holt, 1993; Cheke \& Tratalos, 2007; Tratalos et al., 2010] and have been reviewed by Pener [1991], Pener and Simpson [2009] and Wang and Kang [2014], amongst others. See also Buhl et al. [2006] for a study of hopper band behavior. Holt and Cheke [1996] presented a model of insects with phase change, which can be used to simulate and predict the insects population dynamics for comparisons with observed data. Cheke et al. [2014] expanded the Holt and Cheke [1996] model by including predation together with carrying capacity and population growth rate changes. Here we present and investigate alternative models of phase switching in locusts and demonstrate that they have a rich and varied suite of dynamics including chaos, periodic solutions and coexistence of multiple attractors. We also investigate the effects on the dynamics of different intensities of random noise.

We begin modeling locust dynamics by utilizing the well-known discrete single-population MoranRicker model [Ricker, 1954; Moran, 1950] as follows:

$$
H(t+1)=H(t) \exp \left[r\left(1-\frac{H(t)}{K}\right)\right],
$$

where $H(t)$ denotes the density of the locust population in generation $t, r$ represents the intrinsic growth rate, and $K$ is the carrying capacity of the environment. Next, we introduce the term $g(H)$ in order to consider the effect of predation, which was proposed for insect outbreak systems using the spruce budworm Choristoneurafumiferana and balsam fir Abiesbalsamea system as an example [Ludwig et al., 1978].

$$
g(H)=\frac{\beta H^{2}}{\alpha^{2}+H^{2}},
$$

where the parameters $\beta$ and $\alpha$ are constants. The form of this term takes the following into account: (1) when the insect density is high enough $(H \rightarrow$ $\infty)$, the predation rate should approach an upper limit $\beta$, and (2) when the insect population is low $(H \rightarrow 0)$, the predator will seek other sources of food $H(g(H) \rightarrow 0)$.

Thus, based on (1) and (2), we have the following basic model in the present study to address the phase change of the locust species, i.e. we have

$H(t+1)=H(t) \exp \left[r\left(1-\frac{H(t)}{K}\right)-\frac{\beta H(t)}{\alpha^{2}+H(t)^{2}}\right]$.

It follows from literature [Drury, 2012] that model (3) is a bistable system, which could exist in two stable steady states for particular parameter 
spaces, as shown in Fig. 1(a). For example, if the intrinsic growth rate of the locust population is relatively low, then it can stabilize at the low density (called a refuge equilibrium); once the intrinsic growth rate increases and exceeds some critical value, the density of the locust population can suddenly jump to a high level which is called a high-density outbreak equilibrium [Ludwig et al., 1978].

There is evidence that the intrinsic growth rate for gregarious populations is larger than that for solitarious populations in both the desert locust [Cheke, 1978] and the African armyworm moth Spodopteraexempta [Cheke, 1995] and, further, Holt and Cheke [1996] assumed that the carrying capacity of solitarious populations is less than that of gregarious populations because the latter expand their geographical range from the recession area into the much larger invasion area (see Fig. 3 of [Cheke \& Tratalos, 2007]). Based on these assumptions, a discrete desert locust population model is proposed by using the Ricker equation with switches in intrinsic growth rate and carrying capacity, which can mimic the phase changes of the desert locust population when a random perturbation is introduced into the carrying capacity.

The marked changes of dynamics of the locust and its phases have been revealed by carefully designed experiments and comparing the results with simulations of a self-propelled particle model [Buhl et al., 2006], which revealed a critical density at which marching locusts will spontaneously and suddenly adopt directed collective motion without external perturbation. In an alternative approach towards defining the locust density threshold for transformations to the gregarious stage, Cisse et al. [2013] used field data from the RAMSES database for Mauritania from 2003 to 2011 to explore relations between locust density, phase status and vegetation, by investigating the influence of vegetation on the gregarization thresholds of the desert locust. They showed that the latter varied from 208 to 1525 adults ha-1, dependent upon vegetation cover and condition. However, it is difficult to explore the gregarization threshold in the field due to the complexity of the parameters involved in the dynamics of the locust population.

Therefore, in order to better understand the effects of a gregarization threshold on locust outbreaks and outbreak frequency, we propose the following models that assume that the dynamics of the locust population are bistable with varying carrying capacity and population growth rate, plus a threshold for the phase switches using methods derived from threshold control strategies for integrated pest management [Tang et al., 2015a; Tang et al., 2015b; Tang \& Liang, 2013; Tang et al., 2008]. Based on locust density threshold control, we redescribe the above model as a discrete switching insect outbreak system as follows:

$$
\left\{\begin{array}{r}
H(t+1) \\
\quad=H(t) \exp \left[r\left(1-\frac{H(t)}{K}\right)-\frac{\beta H(t)}{\alpha^{2}+H(t)^{2}}\right], \\
\text { when } H(t)<D T, \\
H(t+1) \\
\quad=H(t) \exp \left[r_{1}\left(1-\frac{H(t)}{K_{1}}\right)-\frac{\beta H(t)}{\alpha^{2}+H(t)^{2}}\right], \\
\text { when } H(t) \geq D T,
\end{array}\right.
$$

where $r<r_{1}, K<K_{1}$ and $D T$ represent the locust density threshold for solitarious to gregarious transformation. This means that the locust population has a low intrinsic growth rate and a low carrying capacity during the solitarious phase, while both the intrinsic growth rate and carrying capacity can significantly increase during the gregarious phase [Holt \& Cheke, 1996]. The two regions $G_{1}$ and $G_{2}$ are defined as follows:

$$
\begin{aligned}
& G_{1}=\{H \mid H<D T, H>0\}, \\
& G_{2}=\{H \mid H \geq D T, H>0\}
\end{aligned}
$$

and consequently we call system (4) defined in region $G_{1}$ as subsystem $S_{G_{1}}$, and in region $G_{2}$ subsystem $S_{G_{2}}$. Our main aim here is to investigate the key factors which can influence the transition from the solitarious phase to the gregarious phase of the locust population, and then address the complex dynamics of model (4).

\section{Equilibrium and Its Stability}

The existences and stabilities of equilibria of both systems $S_{G_{1}}$ and $S_{G_{2}}$, and, in particular, the bistabilities play key roles in determining the switching patterns and complex dynamics of model (4). 


\subsection{Equilibria of the subsystems}

Letting $H_{t}=H_{t+1}=H_{*}$, then the equilibrium $H_{*}$ of subsystem $S_{G_{1}}$ satisfies the following equation

$$
H_{*}=H_{*} \exp \left(r\left(1-\frac{H_{*}}{K}\right)-\frac{\beta H_{*}}{\alpha^{2}+H_{*}^{2}}\right) .
$$

Rearranging the above equation (5), yields

$$
r\left(1-\frac{H_{*}}{K}\right)=\frac{\beta H_{*}}{\alpha^{2}+H_{*}^{2}},
$$

and solving it with respect to $r$ we have

$$
r=\frac{\beta H_{*} K}{\left(\alpha^{2}+H_{*}^{2}\right)\left(K-H_{*}\right)} .
$$

In order to determine the number of equilibria of model (4) [i.e. the positive roots of Eq. (7)] numerically, we choose the intrinsic growth rate as a bifurcation parameter and fix all others as given in the legend for Fig. 1. The equilibrium bifurcation curve with respect to $r$ is shown in Fig. 1(a), from which we can see that the density of the locust population increases as $r$ increases along the path $D B^{\prime} A(r \in(0,0.8042))$ and $B A^{\prime} C(r \in(0.4887$, $1.15)$ ), and there are two stable equilibria for all $r$ in the interval $A B(r \in(0.4487,0.9042))$ and in this region the bistability occurs. The bistability phenomenon provides one explanation of how locust populations switch from a low-density, refuge equilibrium, to a high-density, outbreak equilibrium once the population density has exceeded a threshold.

There are five different patterns for the equilibrium points as $r$ increases. In order to explain the different equilibrium points in more detail, we plotted the left- and right-hand sides of Eq. (6) separately, with the equilibrium points being where the two graphs intersect [see Figs. 1(b)-1(d)].

- Case (i). $0.4887<r<0.9042$

There exist three equilibrium points as shown in Fig. 1(b), which lie on the curve $B^{\prime} A B A^{\prime}$ in Fig. 1(a). $H_{* 1}$ is a stable refuge equilibrium point,

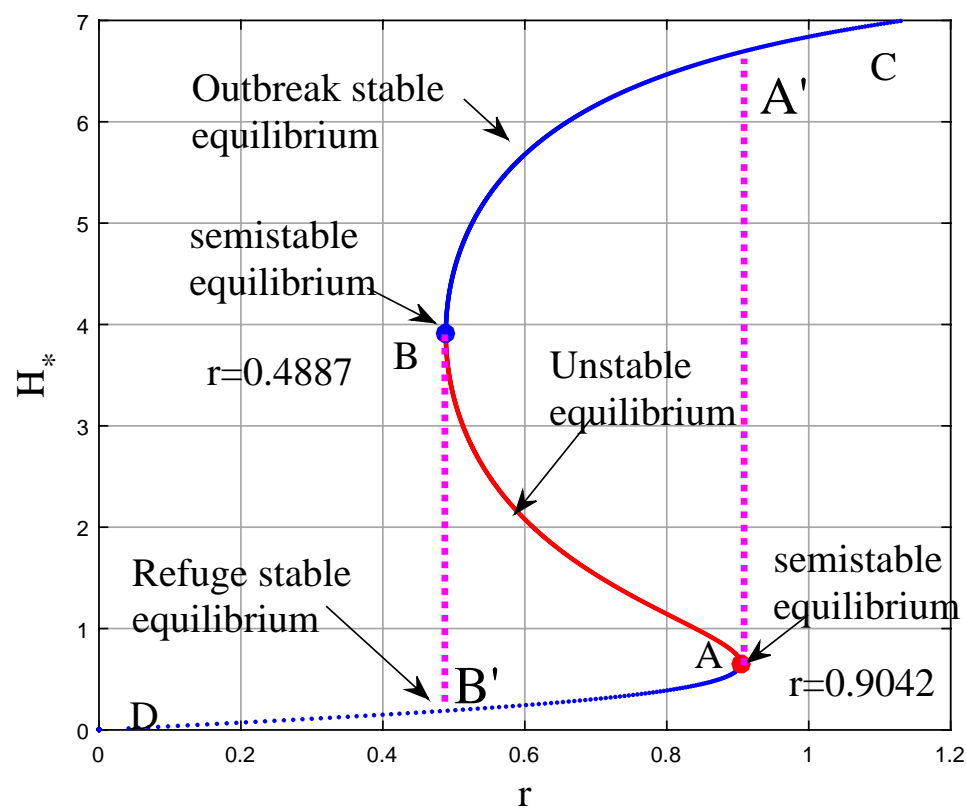

(a)

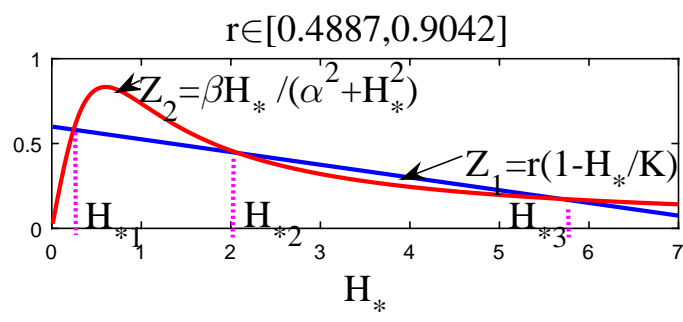

(b)

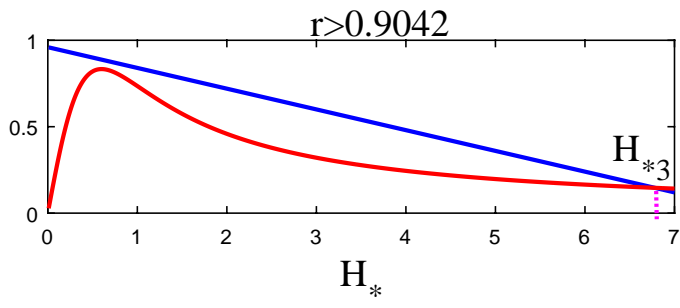

(c)

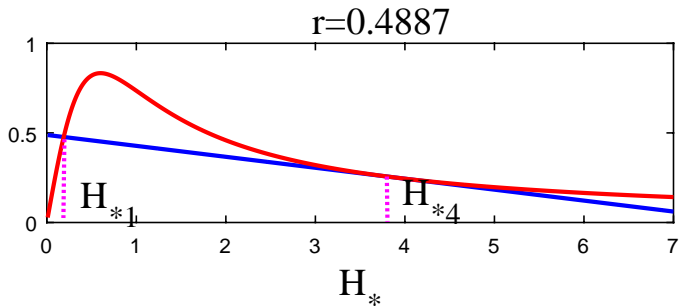

(d)

Fig. 1. Equilibrium points of system (3). (a) Bifurcation curve of equilibrium points with respect to $r$. (b)-(d) The existence of equilibria for different values of parameter $r$. The other parameters are fixed as follows: $\beta=1, K=8, \alpha=1$. 
$H_{* 3}$ is a stable outbreak equilibrium point, and $H_{* 2}$ is an unstable equilibrium point.

- Case (ii). $r>0.9042$

Figure 1(c) shows that only one equilibrium point can exist and it is a stable outbreak equilibrium point.

- Case (iii). $r=0.9042$

There are two equilibrium points [see Fig. 1(d)]. $H_{* 1}$ is a refuge stable equilibrium point, $H_{* 4}$ is a semistable equilibrium point. Moreover, $H_{* 4}$, where the two graphs are tangent to one another, is a bifurcation point.

- Case (iv). $r=0.4887$

Two equilibrium points coexist, one is the outbreak equilibrium point, the other is a point of tangency, a semi-stable equilibrium point.

- Case (v). $0<r<0.4887$

Only the stable refuge equilibrium point exists.

The existence and stability of equilibrium of subsystem $S_{G_{2}}$ can be discussed similarly, so we omit them here.

\subsection{Equilibria for the switching system}

Recently, switching systems have been studied extensively, and differential or difference equations with discontinuous right-hand sides are known as Filippov systems or piecewise smooth systems determined by different equations according to which regions of phase space the solutions pass through [Filippov \& Arscott, 1988]. The classification and stability of all types of equilibria of these systems have been defined and investigated in [Glendinning, 2015; Dercole et al., 2011; Kuznetsov et al., 2003; Xiao et al., 2013; Tang et al., 2012]. In order to investigate the dynamics of locust populations and address how they change between the solitarious phase and the gregarious phase, we define the regular equilibrium and virtual equilibrium for the discrete switching system (4) based on the references [Glendinning, 2015; Dercole et al., 2011; Kuznetsov et al., 2003].

Definition 2.1. An equilibrium point $H_{*}$ is called a regular equilibrium of system (4) if $H_{*}$ is an equilibrium of subsystem $S_{G_{2}}$ and $H_{*}<D T$; or if $H_{*}$ is an equilibrium of subsystem $S_{G_{1}}$ and $H_{*} \geq D T$.
These equilibria will be denoted by $E_{S_{G_{2}}}^{r}$ and $E_{S_{G_{1}}}^{r}$, respectively. A point $H_{*}$ is called a virtual equilibrium of system (4) if $H_{*}$ is an equilibrium of subsystem $S_{G_{2}}$ and $H_{*} \geq D T$; or if $H_{*}$ is an equilibrium of subsystem $S_{G_{1}}$ and $H_{*}<D T$. These equilibria will be denoted by $E_{S_{G_{2}}}^{v}$ and $E_{S_{G_{1}}}^{v}$, respectively.

Note that the stabilities of equilibrium points of system (3) depend on all of the parameters $r, K, \beta, \alpha$, and the positions of all equilibria of model (4) related to the regions $G_{1}$ and $G_{2}$ are quite important for investigations of the dynamics. Thus, we choose the $r$ and $K$ as the bifurcation parameters and fix $\beta$ and $\alpha$, and plot the bifurcation diagram in $r-K$ parameter space for model (3) in Fig. 2(a). There exist three different regions including two monostable regions and a bistable region. The monostable regions are region I (outbreak region marked with purple) and region II (refuge region marked with cyan), the bistable region (i.e. region III) is marked as red with three different equilibrium points in it.

To show the effects of $r_{1}$ and $K_{1}$ on the equilibria of switching system (4), we choose $r_{1}=r+0.1$ and $K_{1}=K+2$ to ensure that $r<r_{1}$ and $K<K_{1}$ in model (4). It follows from Fig. 2(b) that the distribution and stability of the equilibrium points for both subsystem $S_{G_{1}}$ and subsystem $S_{G_{2}}$ have been clearly provided. Where the green area is the unstable region, and the red point is a critical $r$ value which can suddenly switch from the refuge equilibrium point to the unstable equilibrium point or vice versa once the value of $r$ slightly changes from that critical value. The blue curve represents the special case with $r=r_{1}$ and $K=K_{1}$, as shown in Fig. 2(b). The effects of different values of $r$ and $K$ (i.e. $r_{1}$ and $K_{1}$ ) on the distributions of equilibria clarify that how to switch the model from the outbreak phase to the refuge phase and to maintain the density of the locust population below the DT threshold are crucial for control purposes.

Therefore, determining the regions of the whole parameter space and identifying the stabilities of all equilibria of model (4) are useful for addressing the switching frequency from outbreak phase to refuge phase, i.e. from gregarious phase to solitarious phase, and then it can help us to design successful control strategies. To do this, we assume that the gregarious phase subsystem $S_{G_{2}}$ stabilizes at the outbreak equilibrium or is bistable, while the solitarious phase subsystem $S_{G_{1}}$ either stabilizes at 


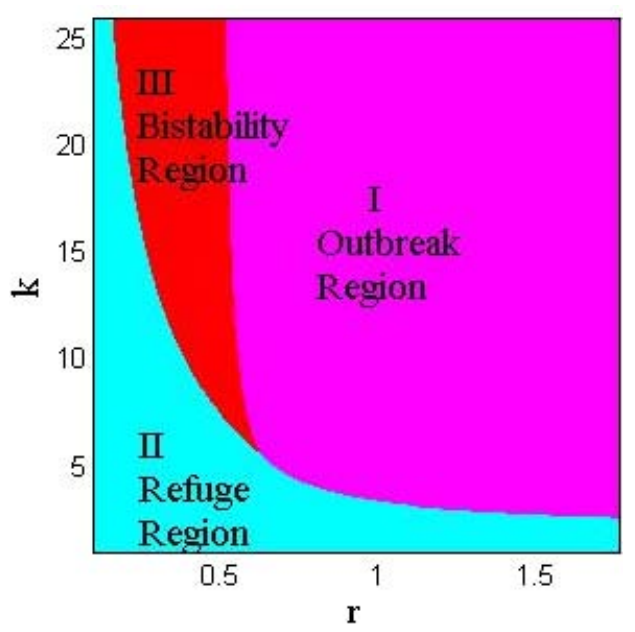

(a)

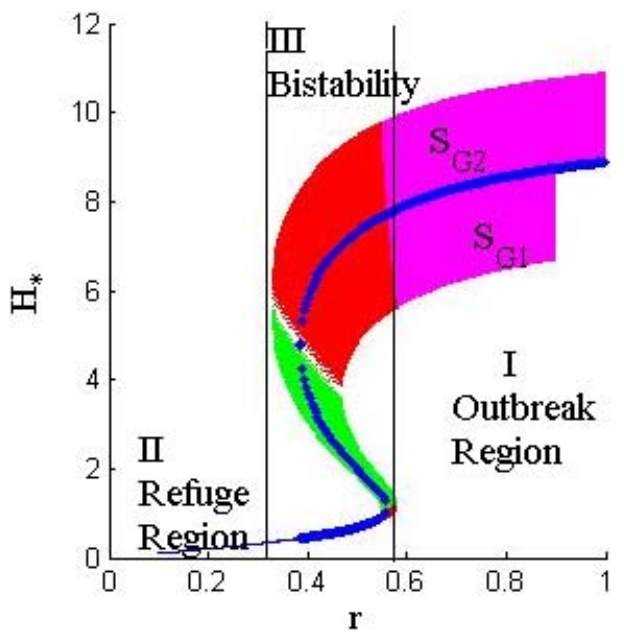

(b)

Fig. 2. Stability regions of equilibrium points. (a) Steady state diagram identifying the regions of parameter space $r-K$ of model (3). The monostability regions are region I (outbreak region marked with purple) and region II (refuge region marked with cyan); the bistability region is region III marked with red. The other parameters are fixed as $\beta=1, \alpha=1$. (b) Comparison of equilibrium points between subsystems $S_{G_{1}}$ and $S_{G_{2}}$. For $S_{G_{2}}$, the parameters $r_{1}$ and $K_{1}$ are changed based on the values of parameters $r$ and $K$, i.e. we have $r_{1}=r+0.1$ and $K_{1}=K+2$ which can ensure that $r<r_{1}$ and $K<K_{1}$.

the refuge equilibrium or is bistable. Furthermore, the stable refuge equilibrium point in subsystem $S_{G_{2}}$ and the outbreak equilibrium for subsystem $S_{G_{1}}$ should be neglected because they are irrelevant to locust outbreaks and the solitarious phase dynamics. Hence, we provide the parameter spaces based on the following four cases:

(1) $S_{G_{2}}$ bistability, $S_{G_{1}}$ refuge;

(2) $S_{G_{2}}$ bistability, $S_{G_{1}}$ bistability;

(3) $S_{G_{2}}$ outbreak, $S_{G_{1}}$ refuge;

(4) $S_{G_{2}}$ outbreak, $S_{G_{1}}$ bistability.

It is difficult to find closed forms for the interior equilibria of these two subsystems, so we employ numerical methods to examine the existence of different types of equilibria and show their coexistence. For system (4), we denoted $E_{R * G 1}$ and $E_{O * G 1}$ as the refuge equilibrium point and the outbreak equilibrium point, respectively, and $E_{B r * G 1}$ and $E_{B o * G 1}$ as bistable equilibrium points in subsystem $S_{G_{1}}$ with $E_{B r * G 1}<E_{B o * G 1}$, which represent the refuge state and outbreak state of the bistability equilibrium points, respectively. Similarly, $E_{R * G 2}, E_{O * G 2}$, $E_{B r * G 2}$ and $E_{B o * G 2}$ represent the different types of equilibria for subsystem $S_{G_{2}}$.

- Case (i). $S_{G_{2}}$ bistability and $S_{G_{1}}$ refuge

In order to investigate the interior equilibria of two subsystems, we choose $r=0.3$ and $K=8$ to ensure a refuge equilibrium point for subsystem $S_{G_{1}}$, and choose $r_{1}=0.5$ and the appropriate $K_{1}$ to give a bistable state in subsystem $S_{G_{2}}$ [see Fig. 2(a)]. Moreover, we choose $k_{1}$ and $D T$ as bifurcation parameters, and the density threshold $D T$ can vary from 0.2 to 10 and the carrying capacity $K_{1}$ can vary from 8.1 to 18.

For Case (i), the parameter space has been divided into four regions, which have been marked in different colors, as shown in Fig. 2(a). This clarifies that the existence and stability of these equilibria depend on the values of $D T$ and $K_{1}$. Note that if the density threshold $D T$ is large enough, then all equilibrium points of the two subsystems are below $D T$ (i.e. $D T>E_{B o * G 2}>E_{B r * G 2}>$ $\left.E_{R * G 1}\right)$, which indicates that both subsystems can switch freely from one to the other [region I in Fig. 3(a)]. Thus, we do not concern ourselves with this region later. Meanwhile, the density threshold line $H=D T$ should be above the regions III and IV [see Fig. 3(a)]. In region IV, the density threshold is relatively small, and we have $E_{R * G 1}>D T$, $E_{B r * G 2}>D T$ and $E_{B o * G 2}>D T$. In region III, we have $E_{R * G 1}<D T, E_{B r * G 2}>D T$ and $E_{B o * G 2}>$ $D T$. Both regions have a low-density threshold, and the refuge state of the bistable equilibrium points in subsystem $S_{G_{2}}$ is greater than $D T\left(E_{B r * G 2}>D T\right)$. This is not realistic and may be irrelevant to locust phase changes and outbreaks, so we only focus on the regions I and II in this case. 


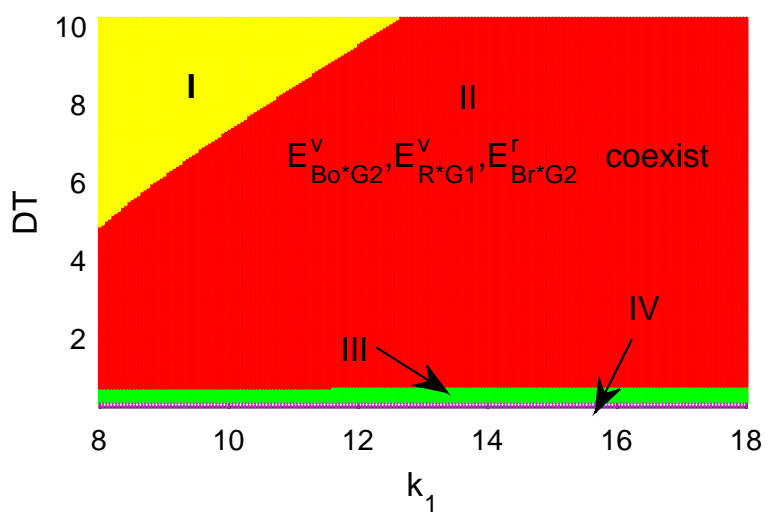

(a)

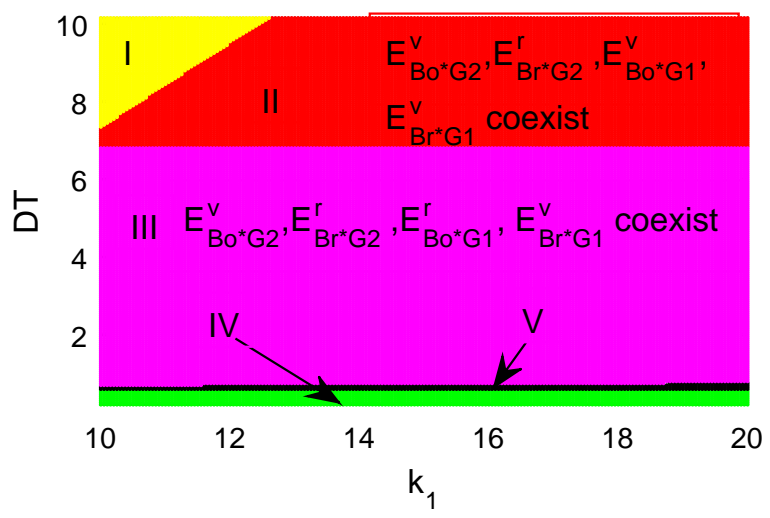

(c)

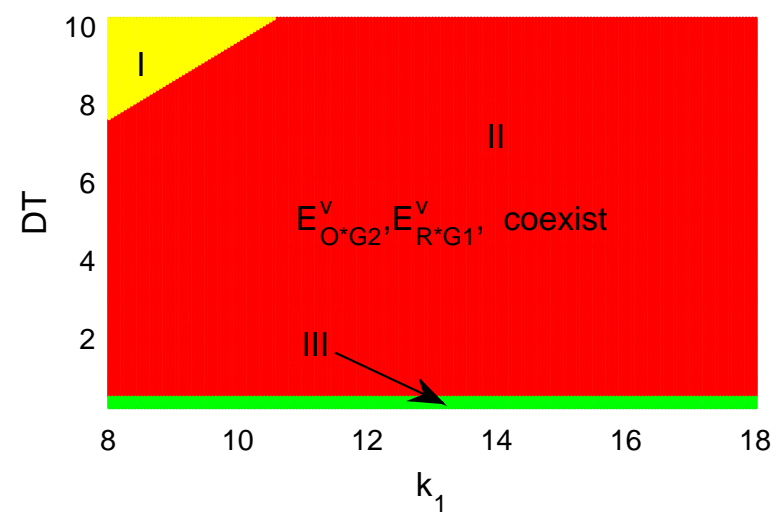

(b)

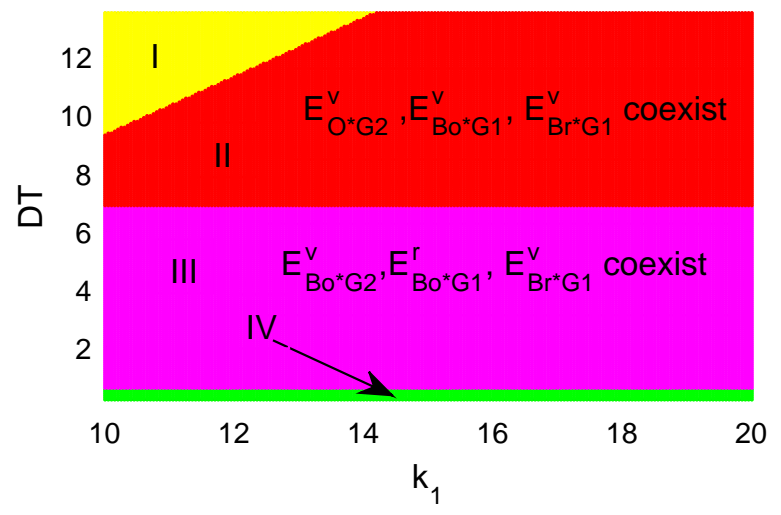

(d)

Fig. 3. Parameter bifurcation diagrams in $D T-K_{1}$ space of (4). (a) Case (i) with $S_{G_{2}}$ bistability and $S_{G_{1}}$ refuge, where $r=0.3, K=8, r_{1}=0.5$. (b) Case (ii) with $S_{G_{2}}$ outbreak and $S_{G_{1}}$ refuge, where $r=0.3, K=8, r_{1}=2.5$. (c) Case (iii) with $S_{G_{2}}$ bistability and $S_{G_{1}}$ bistability, where $r=0.45, K=10, r_{1}=0.5$. (d) Case (iv) with $S_{G_{2}}$ outbreak and $S_{G_{1}}$ bistability, where $r=0.45, K=10, r_{1}=1.5$. The other parameters are fixed as $\beta=1, \alpha=1$.

It follows from the region II of Fig. 3(a) that $E_{B o * G 2}>D T>E_{B r * G 2}>E_{R * G 1}$, and we denote the corresponding equilibria as $E_{B o * G 2}^{v}$, $E_{B r * G 2}^{r}, E_{R * G 1}^{v}$ according to Definition 2.1. Moreover, $E_{B o * G 2}^{v}, E_{R * G 1}^{v}$ and $E_{B r * G 2}^{r}$ can coexist in region II (red) [as shown in Fig. 3(a)], which could result in changing locust phases from the solitarious to the gregarious.

Therefore, for relatively larger density thresholds $D T$ (for example $H(t)=D T>E_{B o * G 2}$ ), the solitarious phase subsystem $S_{G_{1}}$ only has the refuge equilibrium [region I in Fig. 3(a)]. Thus, we have $E_{B o * G 2}>D T>E_{B r * G 2}>E_{R * G 1}$ [see Fig. 3(a)], and we denote the corresponding equilibria as $E_{B o * G 2}^{v}, E_{R * G 1}^{v}, E_{B r * G 2}^{r}$ according to Definition 2.1. Moreover, $E_{B o * G 2}^{v}, E_{R * G 1}^{v}$ and $E_{B r * G 2}^{r}$ can coexist in region II (red) [as shown in Fig. 3(a)], which could result in transformation of the locust phases from the solitarious to the gregarious. Thus, for this case we have, based on Fig. 3(a)

region I, $D T>E_{B o * G 2}>E_{B r * G 1}>E_{R * G 1}$, $E_{B o * G 2}^{r}, E_{B r * G 2}^{r}, E_{R * G 1}^{v}$ can coexist;

region II, $E_{B o * G 2}>D T>E_{B r * G 1}>E_{R * G 1}$, $E_{B o * G 2}^{v}, E_{B r * G 2}^{r}, E_{R * G 1}^{v}$ can coexist;

region III, $E_{B o * G 2}>E_{B r * G 1}>D T>E_{R * G 1}$, $E_{B o * G 2}^{v}, E_{B r * G 2}^{v}, E_{R * G 1}^{v}$ can coexist;

region IV, $E_{B o * G 2}>E_{B r * G 1}>E_{R * G 1}>D T$, $E_{B o * G 2}^{v}, E_{B r * G 2}^{v}, E_{R * G 1}^{r}$ can coexist.

- Case (ii). $S_{G_{2}}$ outbreak and $S_{G_{1}}$ refuge

This case can be discussed in a similar way as for Case (i), as shown in Fig. 3(b). In particular, choosing $r_{1}=2.5$ can ensure that subsystem $S_{G_{2}}$ has 
only an outbreak equilibrium point. There exist three regions in $K_{1}-D T$ parameter space and region II has two virtual equilibria $E_{O * G 2}^{v}$ and $E_{R * G 1}^{v}$ which can coexist, in conclusion we have

region I, $D T>E_{O * G 2}>E_{R * G 1}, E_{O * G 2}^{r}$ and $E_{R * G 1}^{v}$ which can coexist;

region II, $E_{O * G 2}>D T>E_{R * G 1}, E_{O * G 2}^{v}$ and $E_{R * G 1}^{v}$ which can coexist;

region III, $E_{O * G 2}>E_{R * G 1}>D T, E_{O * G 2}^{v}$ and $E_{R * G 1}^{r}$ which can coexist.

- Case (iii). $S_{G_{2}}$ bistability and $S_{G_{1}}$ bistability

Both subsystems have bistable states in this situation, where $r=0.45, k=10, r_{1}=0.5$. Figure 3(c) shows that there exist five different regions. In region II (red) and region III (purple) all $E_{B o * G 2}^{v}, E_{B r * G 2}^{r}, E_{B o * G 1}^{v}, E_{B r * G 1}^{v}$ and $E_{B o * G 2}^{v}$, $E_{B r * G 2}^{r}, E_{B o * G 1}^{r}, E_{B r * G 1}^{v}$ can coexist, and we have

region I, $D T>E_{B o * G 2}>E_{B o * G 1}>E_{B r * G 2}>$ $E_{B r * G 1}, \quad E_{B o * G 2}^{r}, \quad E_{B r * G 2}^{r}, \quad E_{B o * G 1}^{v}, \quad E_{B r * G 1}^{v}$ can coexist;

region II, $E_{B o * G 2}>D T>E_{B o * G 1}>E_{B r * G 2}>$ $E_{B r * G 1}, \quad E_{B o * G 2}^{v}, \quad E_{B r * G 2}^{r}, \quad E_{B o * G 1}^{v}, \quad E_{B r * G 1}^{v}$ can coexist;

region III, $E_{B o * G 2}>E_{B o * G 1}>D T>E_{B r * G 2}>$ $E_{B r * G 1}, \quad E_{B o * G 2}^{v}, \quad E_{B r * G 2}^{r}, \quad E_{B o * G 1}^{r}, \quad E_{B r * G 1}^{v}$ can coexist;

region $\mathrm{IV}, E_{B o * G 2}>E_{B o * G 1}>E_{B r * G 2}>D T>$ $E_{B r * G 1}, \quad E_{B o * G 2}^{v}, \quad E_{B r * G 2}^{v}, \quad E_{B o * G 1}^{r}, \quad E_{B r * G 1}^{v}$ can coexist;

region $\mathrm{V}, E_{B o * G 2}>E_{B o * G 1}>E_{B r * G 2}>E_{B r * G 1}>$ $D T, E_{B o * G 2}^{v}, E_{B r * G 2}^{v}, E_{B o * G 1}^{r}, E_{B r * G 1}^{r}$ can coexist.

- Case (iv). $S_{G_{2}}$ outbreak and $S_{G_{1}}$ bistability

Subsystem $S_{G_{2}}$ has only outbreak equilibrium point with $r_{1}=1.5$. There are four different regions in Fig. 3(d). In region II, $E_{O * G 2}^{v}, E_{B o * G 1}^{v}, E_{B r * G 1}^{v}$ can coexist and in region III, $E_{O * G 2}^{v}, E_{B o * G 1}^{r}, E_{B r * G 1}^{v}$ can also coexist. So we have

region I, $D T>E_{O * G 2}>E_{B o * G 1}>E_{B r * G 1}, E_{O * G 2}^{r}$, $E_{B o * G 1}^{v}, E_{B r * G 1}^{v}$ can coexist;

region II, $E_{O * G 2}>D T>E_{B o * G 1}>E_{B r * G 1}$, $E_{O * G 2}^{v}, E_{B o * G 1}^{v}, E_{B r * G 1}^{v}$ can coexist;

region III, $E_{O * G 2}>E_{B o * G 1}>D T>E_{B r * G 1}$, $E_{O * G 2}^{v}, E_{B o * G 1}^{r}, E_{B r * G 1}^{v}$ can coexist; region IV, $E_{O * G 2}>E_{B o * G 1}>E_{B r * G 1}>D T$, $E_{O * G 2}^{v}, E_{B o * G 1}^{r}, E_{B r * G 1}^{r}$ can coexist.

Although control measures should be applied once the density of the locust population reaches the threshold density $D T$ (i.e. control tactics should be involved in subsystem $S_{G_{2}}$ ), we mainly focus on the switching frequency and the complex dynamics of model (4) without control strategies in the coming sections. Model (4) with chemical and biological control tactics will be studied in the near future. Based on the analyses of the above cases, the interesting regions which will be investigated in more detail are regions II in Figs. 3(a)-3(d) and regions III in Figs. 3(c) and 3(d). Note that the equilibria $E_{O * G 2}^{v}$ or $E_{B o * G 2}^{v}$ exist in these regions.

\section{Numerical Investigation of Model (4) with Density Threshold}

In this section, we describe numerical investigations of system (4) by employing bifurcation analysis, heatmap and random perturbation methods. In particular, we determine the key factors which can reveal the existence of multiple attractors, coexistence, initial sensitivity and switching behavior.

\subsection{Bifurcation analysis and complex behavior}

In order to gain preliminary insights into the properties of the dynamical system, we first give the onedimensional bifurcation diagram with respect to the intrinsic growth rate $r$ such that $r<r_{1}$ for fixed $r_{1}$. Note that for the parameter set given in Fig. 4 we can see that both subsystems $S_{G_{1}}$ and $S_{G_{2}}$ have outbreak equilibrium points.

We first choose $r$ as the bifurcation parameter and fix all other parameters as follows: $D T=$ $3.5, \beta=1.1, K=4, \alpha=0.1, r_{1}=3.5, K_{1}=5.6$ and initial values $H_{0}=0.5$. It follows from Fig. 4(a) that the switching system can exhibit very complex dynamics as the intrinsic growth rate increases. Compared with the Ricker model without switching between two subsystems, we found that the switching could result in more complex bifurcation behavior, as shown in Fig. 4(a), from which we can see that the periodic, quasi-periodic, chaotic solutions and Hopf or period-doubling bifurcations occur frequently as the parameter varies. For example, as the parameter $r$ increases from 2.1 


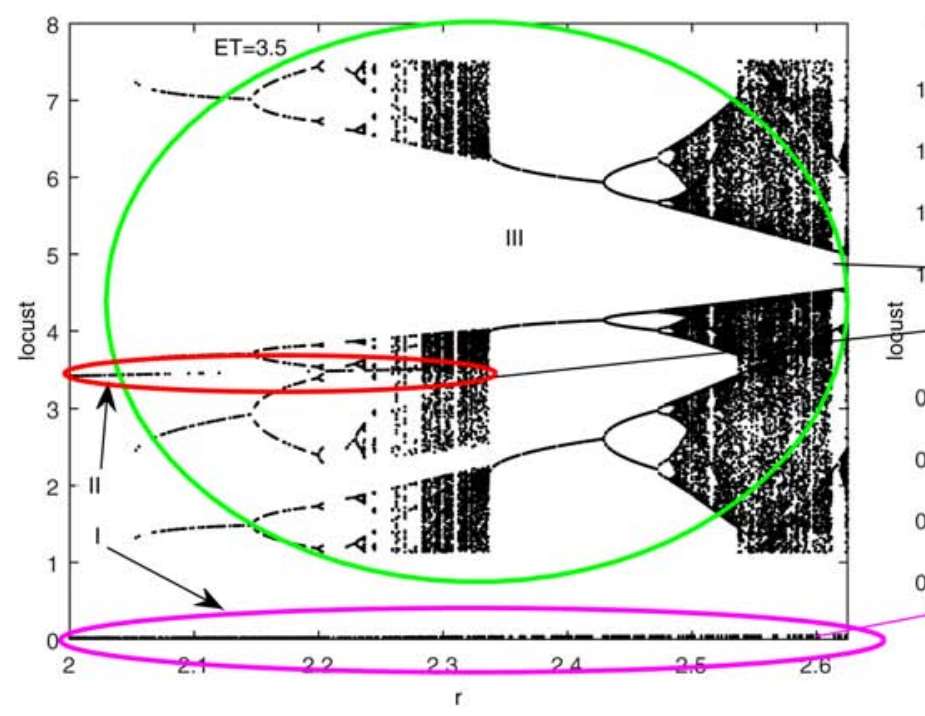

(a)

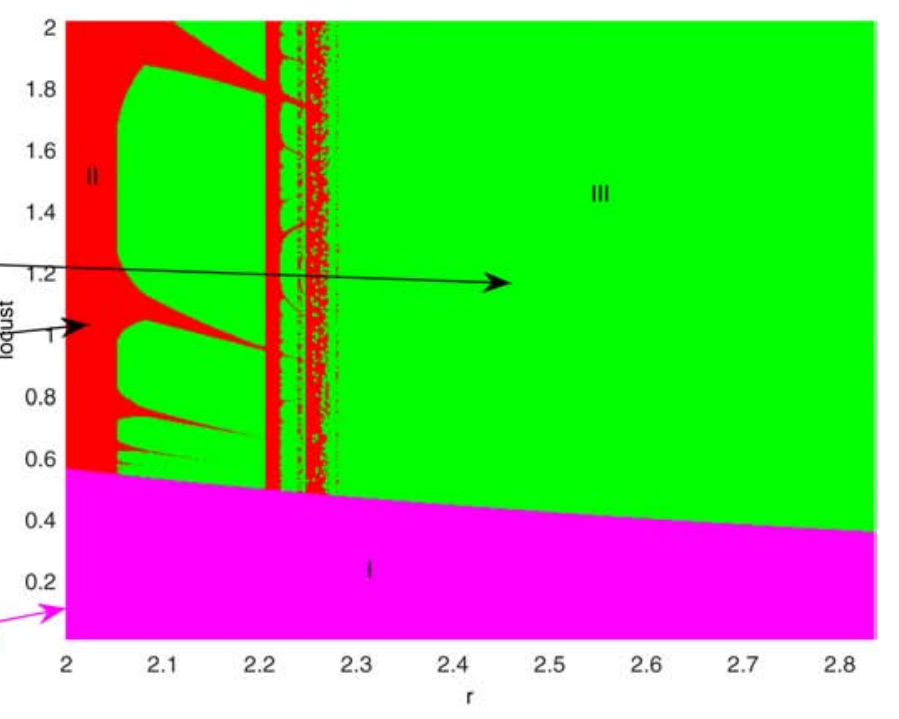

(b)

Fig. 4. Bifurcation diagrams of model (4). (a) Parameter bifurcation diagram. The other parameters are fixed as $D T=$ $3.5, \beta=1.1, K=4, \alpha=0.1, r_{1}=3.5, K_{1}=5.6$, the initial value $H_{0}=0.5$. (b) Initial value regions with respect to different values of $r$ for (a).

to 2.2 , the system's behavior suddenly changes to period-doubling bifurcations, and a chaotic attractor abruptly appears at $r=2.3$. Once the bifurcation parameter $r$ exceeds the threshold value around 2.35 , then a period-halving bifurcation occurs and another chaotic attractor emerges abruptly around $r=2.5$.

There exist three different types of attractors which are denoted as regions I-III for a wide range of values for parameter $r$ [as shown in Fig. 4(b)]. The attractor $\mathrm{I}$ is close to a refuge equilibrium point with quite low values such as 0.05 , which is much lower than the density threshold DT $(D T=3.5)$. Note, especially, that this attractor can coexist for a large range of $r$ with both attractors II and III. When $r$ is increased from 2 to 2.35 , we find that the attractor I can coexist with attractor II and this attractor is stable and below DT. The stable attractor III is completely determined by the switching system (4) which switches around DT when $r$ is increased from 2.05 to 2.6. The stable attractor III indicates that the locust phases can change from solitarious to gregarious forever, and consequently outbreaks of the locust population occur frequently.

In order to explain the effects of the initial values on those three attractors in more detail, the basins of attraction of the initial values with different $r$ values are presented in Fig. 4(b). There exist three different regions corresponding to Fig. 4(a). The region I (purple) is the basin of attraction region of attractor $\mathrm{I}$, and the initial values are less than 0.6. Moreover, as the parameter $r$ increases, the basin of attraction region becomes smaller. The region II (red) is the basin of attraction region of attractor II and the region III (blue) corresponds to the same for attractor III. It follows from regions II and III that the intrinsic growth rate and the initial values of the locust population are sensitive, which may result in the outbreak of locust populations and phase switching once a small perturbation occurs. The questions are: (1) How frequently does switching occur from solitarious to gregarious and vice versa; (2) When does the transition occur such that the solutions with larger amplitudes from region III can switch to a stable attractor in region II? and (3) How does the random perturbation influence the switching pattern? We will discuss these issues in more detail in the following sections.

\subsection{Switching transients for phase change}

The analysis in Sec. 3.1 showed that different attractors can coexist for a wide range of values for the intrinsic growth rate $r$. What we want to know is whether switching could occur among different attractors as initial values vary, i.e. how do the switch-like transitions change as parameter values and initial values vary? In this context, it is interesting to note that studies on lakes, coral reefs, 
oceans, forests and arid lands have shown that smooth changes can be interrupted by sudden drastic switches to a contrasting state [Scheffer et al., 2001]. To address such switching behavior, we define switching transients as follows:

Definition 3.1. If the attractors switch from one attractor to another, then this switch-like transitional change is called the switching transient. Also, the generation at which the attractor III can shift to the stable attractor II is called a stable transient point.

To compute the stable transient point for system (4) conveniently and realistically, we only calculate the stable transient point within 5000 generations. Therefore, we set the switching transient point to be 5000 but if the phase change does not occur within 5000 generations, and if the initial values lie in attractor I and are much lower than DT, then the phase change will never occur and consequently we denote its transient point as 0 .

The color map in Matlab was employed to show how fast the switching occurs, as shown in Fig. 5(a), where the highest stable transient point is indicated by a warm (red) color whereas the lowest stable transient point is indicated by a cold (blue) color. Note that when the initial values lie around the intervals $[0,0.5]$ and $[9.1,9.8]$, the locust populations do not change their phase at all and according to the definition the attractor switching point is 0 . Two examples are shown in Figs. 5(d) and 5(g).

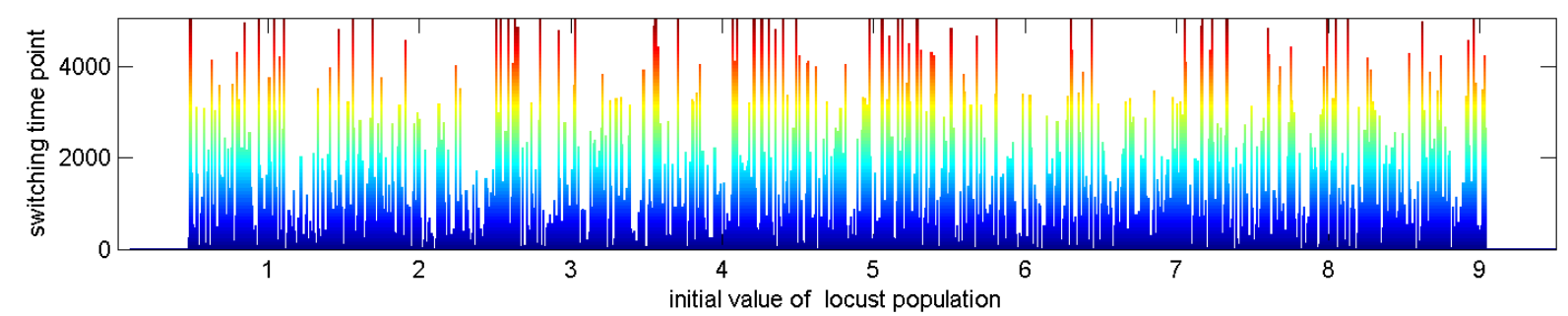

(a)

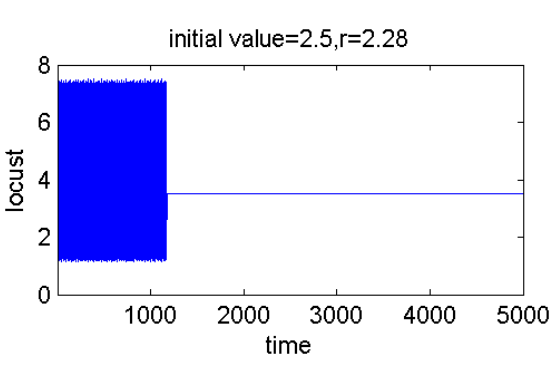

(b)

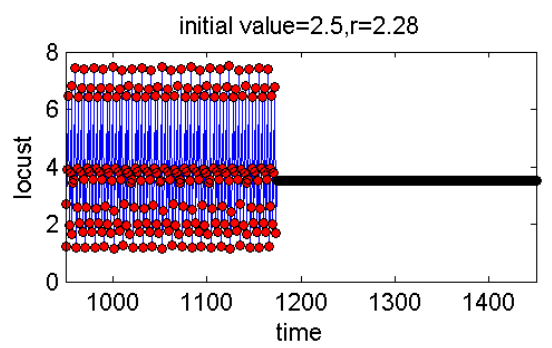

(e)

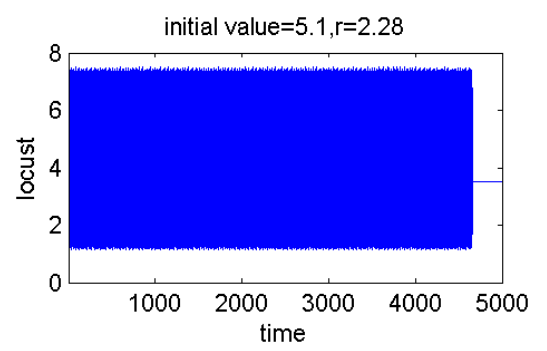

(c)

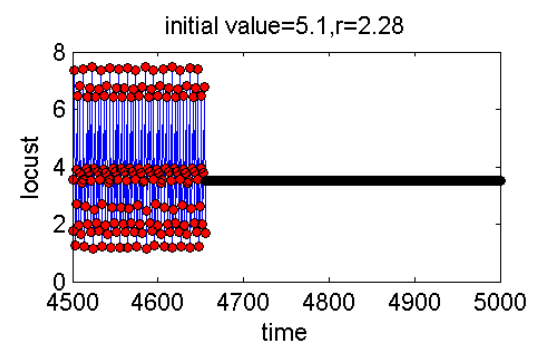

(f)

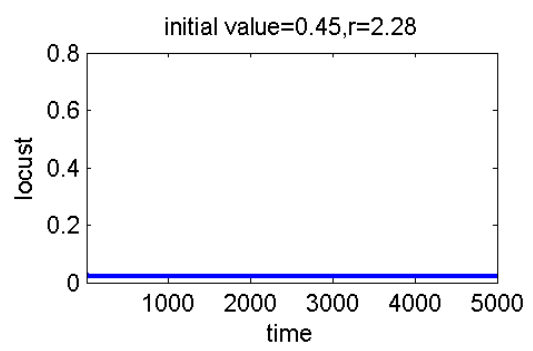

(d)

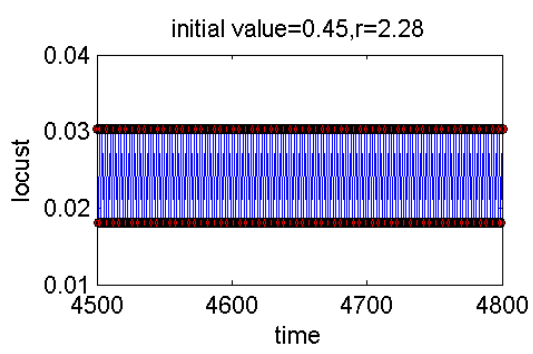

(g)

Fig. 5. Switching transients of model (4). (a) Stable transient point over 5000 generations as the initial value varies from 0 to 9.8. (b) The time series with initial value $=2.5$. (c) The time series with initial value $=5.1$. (d) The time series with initial value $=0.45$. (e) $-(\mathrm{g})$ are enlargements of $(\mathrm{b})-(\mathrm{d})$, respectively. The other parameters are fixed as follows: $D T=3.5, \beta=1.1, K=4, \alpha=0.1, r_{1}=3.5, K_{1}=5, r=2.28$. 
It is obvious that the switching transient exists in Figs. 5(b) and 5(c). Figures 5(e) and 5(f) are enlargements of Figs. 5(b) and 5(c) with initial values 2.5 and 5.1, respectively. Moreover, the stable transient point occurs at the 1172-th generation in Fig. 5(e), and the stable transient point in Fig. 5(f) is at the 4652-th generation. Note that the final states shown in Figs. 5(b) and 5(c) indicate that the densities of the locust population are less than DT. If so, the switching transient may be beneficial for locust control in this case.

However, the switching transients may be harmful once multiple attractors can coexist, as shown in Figs. 6(b)-6(d). The switch-like transitions occur frequently among different attractors, which depend on the intrinsic growth rate $r$ and initial values. Morever, there is almost no stable transient point for the intrinsic growth rate $r=2.9$ and the wide range of initial values (here from 0.5 to 9.8), as shown in Fig. 6(a). The frequent switching transients indicate that the pest population can have outbreaks at different scales. Note that when the initial values lie in the interval $[0,0.5]$, the locust population does not change its phase at all.

The sensitivity of the stable transient point with respect to the parameters of the intrinsic growth rate $r$, initial values and the threshold density $D T$ are crucial for successful pest control. In order to show this in more detail, by employing the heat-map we address switching transients numerically as shown in Fig. 7. The largest stable transient point is indicated by a warm (red) color whereas the

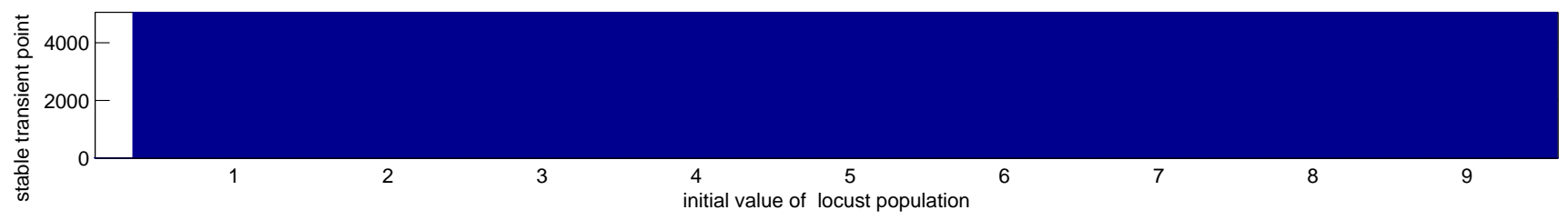

(a)

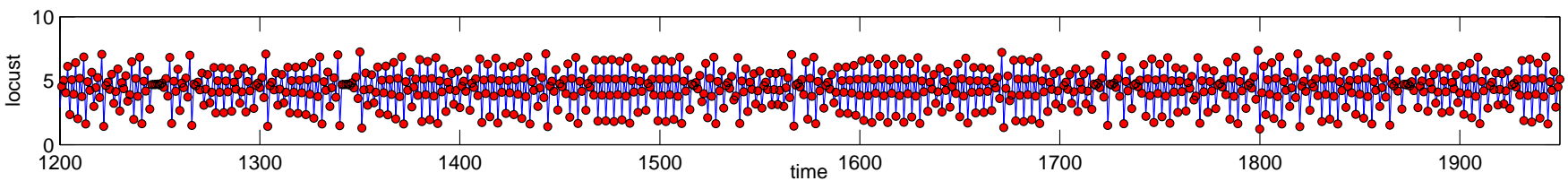

(b)

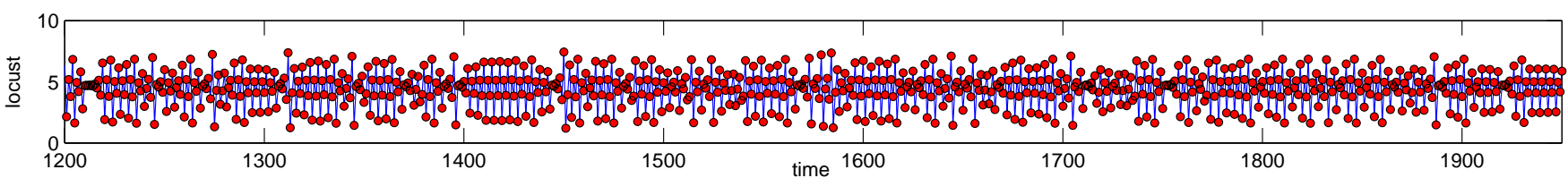

(c)

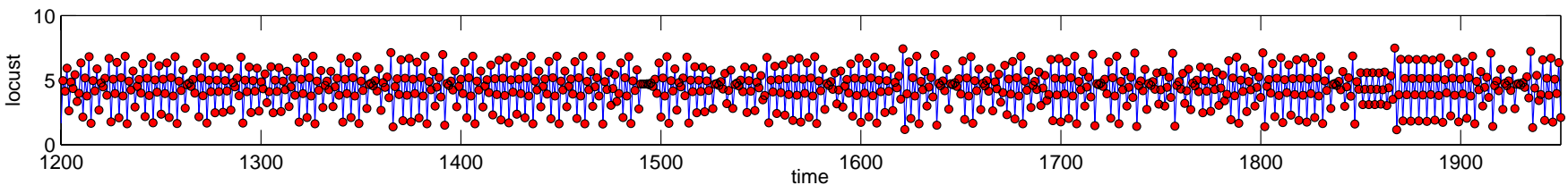

(d)

Fig. 6. Switching transients of model (4) with $r=2.9$. (a) Stable transient point over 5000 generations as the initial value varies from 0 to 9.8. (b) The time series with initial value $=2.5$. (c) The time series with initial value $=5.1$. (d) The time series with initial value $=0.45$, respectively. The other parameters are fixed as $D T=3.5, \beta=1.1, K=4, \alpha=0.1, r_{1}=3.5, K_{1}=$ $5, r=2.9$. 


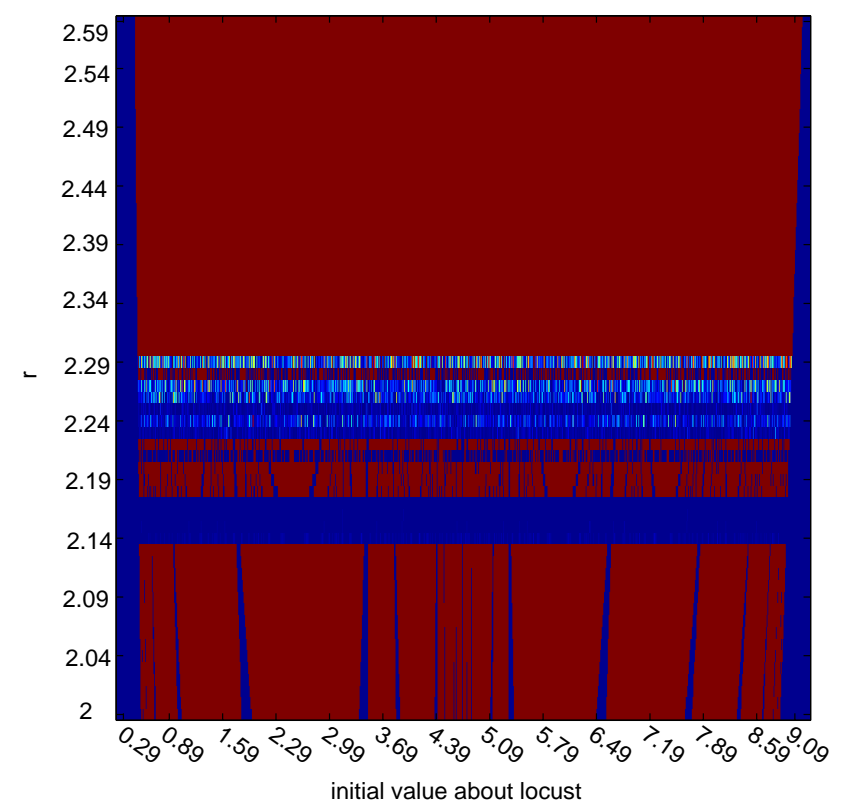

(a)

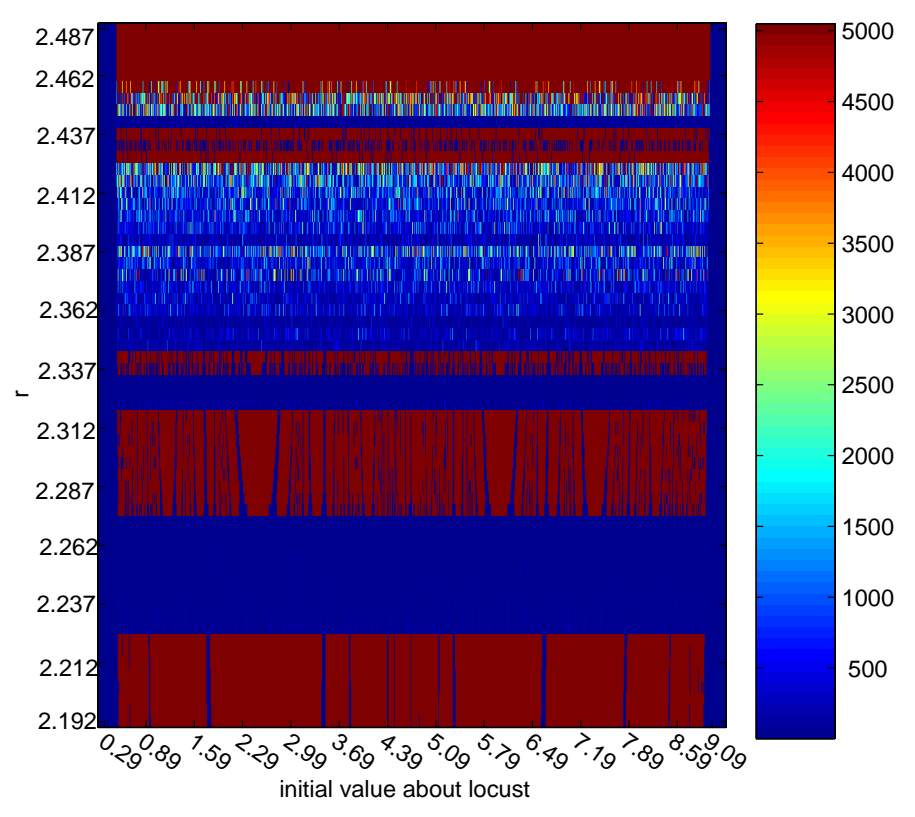

(b)

Fig. 7. Heat map of switching transients with different values of $r$ and initial values. The threshold density $D T=3.5$ for (a) and $D T=4$ for (b). The other parameters are fixed as follows: $\beta=1.1, \alpha=0.1, K=4, K_{1}=5, r_{1}=3.5$.

smallest stable transient point is indicated by a cold (blue) color. It follows from the heat-map shown in Fig. 7 that the intrinsic growth rate, initial values and the threshold density DT can significantly influence the stable transient points. For example, in Fig. 7(a) with $D T=3.5$, when $r$ belongs to the interval $(2.24,2.29)$, the stable transient points vary frequently. For a different threshold density $D T$ the results shown in Fig. 7(b) indicate that the stable transient points vary even more frequently when $r \in(2.36,2.462)$. Note that the two stable attractors must coexist for $r \in(2.14,2.18)$ in (a) and $r \in(2.24,2.27)$ in (b). The changes of the stable transient points also indicate that the locust population could switch irregularly, which may result in complexity for the implementation of control measures.

\subsection{The effects of random perturbation on the stable transient point}

Locust populations are often subject to the effects of environmental fluctuations. For models with a bistable deterministic skeleton, this means that understanding when and how stochastic events will promote or inhibit attractor switching transients, requires careful study of a stochastic version of the model [Guttal \& Jayaprakash, 2007; Serizawa et al., 2009]. In order to investigate the locust population's bistable dynamics with random perturbation, we redescribe the discrete switching insect outbreak model in the following text, aiming to address how stochastically forced attractor switching occurs and the shifting of two attractors.

$$
\left\{\begin{array}{l}
\left.H(t+1)=H(t) \exp \left[r\left(1-\frac{H(t)}{K}\right)-\frac{\beta H(t)}{\alpha^{2}+H(t)^{2}}\right]+\tau_{t}\right\} \quad \text { when } H(t)<D T ; \\
\left.H(t+1)=H(t) \exp \left[r_{1}\left(1-\frac{H(t)}{K_{1}}\right)-\frac{\beta H(t)}{\alpha^{2}+H(t)^{2}}\right]+\tau_{t}\right\} \quad \text { when } H(t) \geq D T,
\end{array}\right.
$$

where $\tau_{t}=0$ means that random perturbation does not exist at generation $t$ and $\tau_{t}=\sigma u$ indicates that there is random perturbation at generation $t$, and $u$ is a uniformly distributed variable on $[0,1]$ and $\sigma>0$ represents the intensity of noise. 
Based on the previous discussion and Fig. 4, we know that attractors I-III can coexist simultaneously for a wide range of parameters such as $r \in(2,2.35)$. It follows from Figs. 5 and $8(\mathrm{a})$ that the solutions can finally stabilize at the attractor II after some generations of oscillations, which means that the density of the locust population can remain below the threshold density $D T$ if the population does not experience any random perturbation. Therefore, what we want to address is how the random perturbation affects the switching behavior of the locust population. To do this, the time series of model (8) with or without random perturbations are shown in Fig. 8. Figure 8(a) shows the system has switching transients and the stable transient point is about 20 (without random perturbation, $\sigma=0)$.

If we choose same intensity of noise which has been generated firstly based on the number of iterations to the solution and different initial values, the results shown in Figs. 8(b) and 8(c) indicate that multiple switchings occur between attractors II and III, which show that the switching patterns could significantly vary for different random perturbation and initial values. Note that an interesting result shown in Figs. 8(b) and 8(c) is that the switching transient patterns become identical after 480 generations (point A, red dashed line), suggesting that the evolving process of the perturbation system (8) is not related to the initial value after some fixed number of generations for the same stochastic processes, which indicates that the density of the population is also the same after a fixed number of iterations.

In the following section, based on Definition 3.1 we will discuss the switching frequency for different noise intensities and different values of $r$. That is, the two interesting questions are: how many attractor switches can exist, and at what generation numbers can the switching system (8) switch?

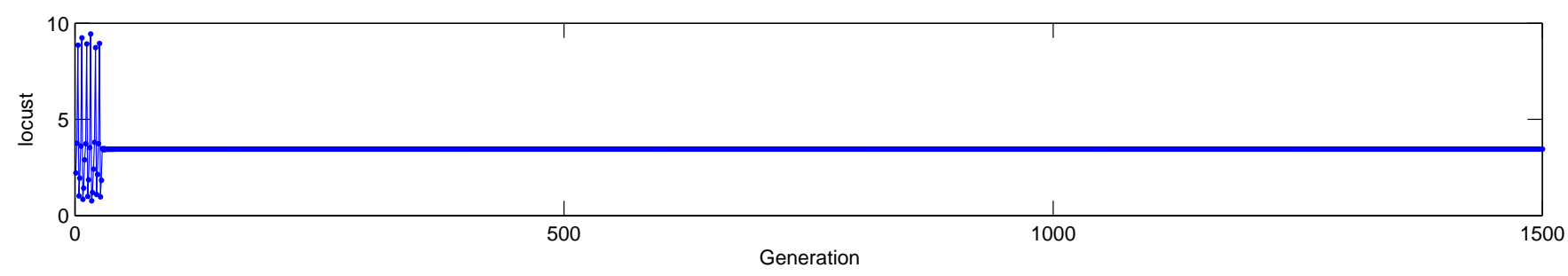

(a)

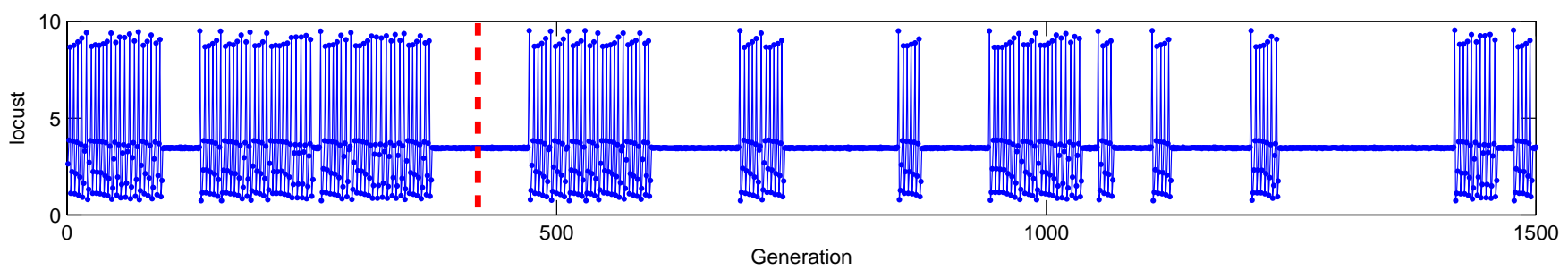

(b)

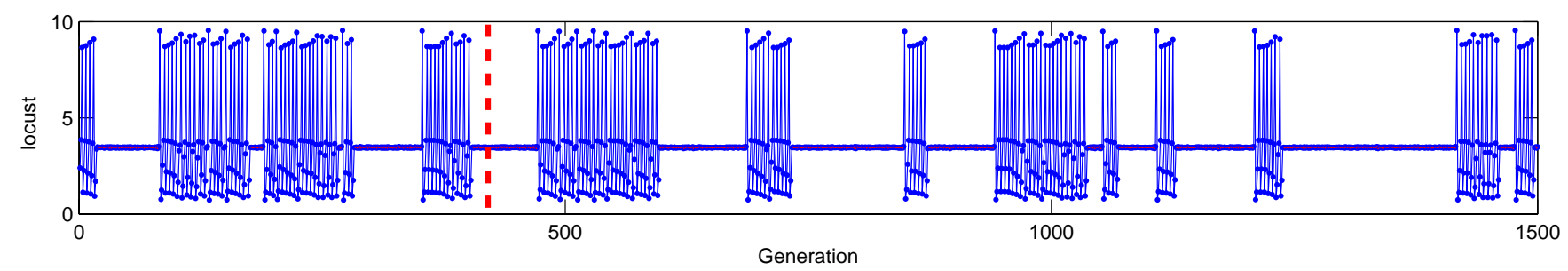

(c)

Fig. 8. The effects of random perturbation on the switching transient for model (8). (a) The intensity of noise $\sigma=0$ and initial value $H_{0}=5.2$. (b) The intensity of noise $\sigma=0.05$ and initial value $H_{0}=5.2$. (c) The intensity of noise $\sigma=0.05$ and initial value $H_{0}=7.2$. The other parameters are fixed as follows: $D T=3.5, \beta=1.1, \alpha=0.1, r=2.28, k=4, r_{1}=3.5, K_{1}=5.6$. 


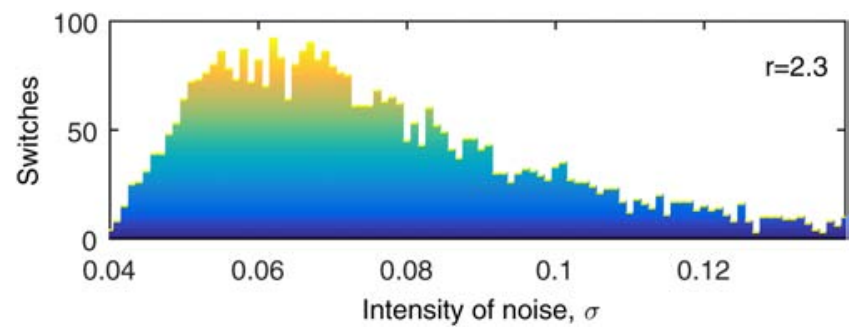

(a)

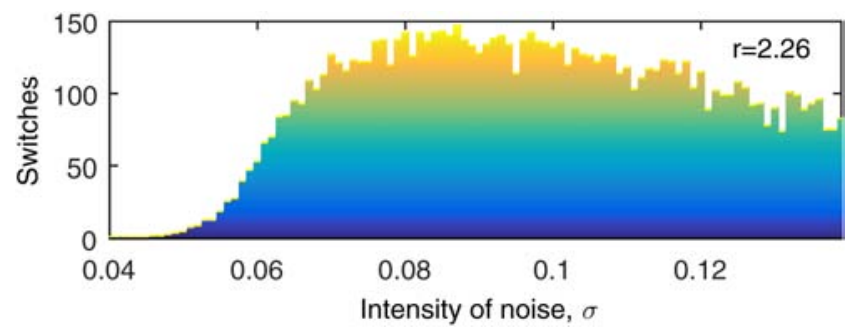

(c)

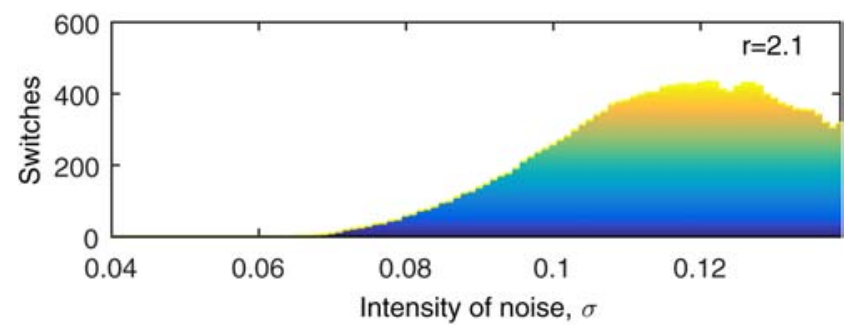

(e)

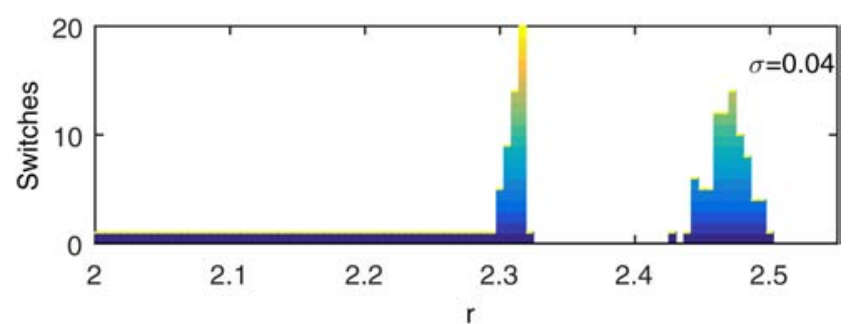

(b)

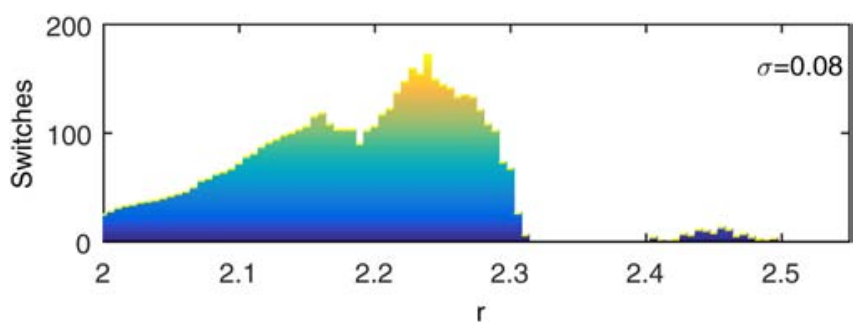

(d)

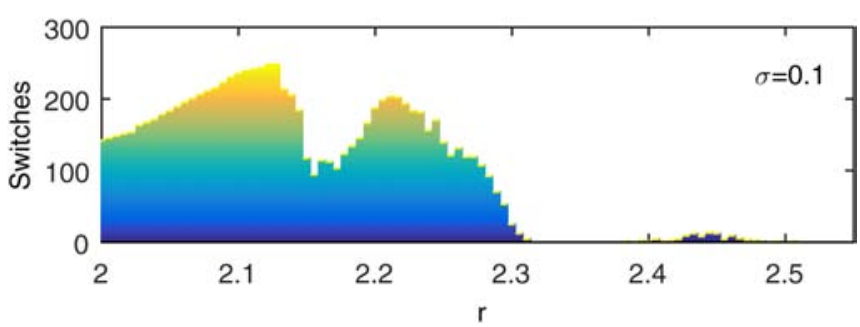

(f)

Fig. 9. Number of stable transient switches for system (8). (a), (c) and (e) with $r=2.3,2.26$ and 2.1, respectively. (b), (d) and (f) with $\sigma=0.04,0.08$ and 0.1 , respectively. The other parameters are fixed as follows: $D T=3.5, \beta=1.1, K=4, \alpha=$ $0.1, r_{1}=3.5, K_{1}=5.6$.

For a better explanation of the influence of the number of attractor switches, we discuss them in the context of different values of the parameter $r$ and intensity of noise $\sigma$. Figure 9 shows different numbers of attractor switches with different parameters. In order to avoid the effects of initial values on the switching patterns [see Figs. 8(b) and 8(c)], we omit the first 1000 generations for each simulation. The numbers of attractor switches were calculated between the 1001-th generation and the 9900-th generation. Firstly, let the parameter $r$ be fixed and the intensity of noise vary from 0.04 to 0.14, Figs. 9(a), 9(c) and 9(e) show different numbers of attractor switches with $r=2.3, r=2.26$ and $r=2.1$, respectively. When $r=2.3$, the maximum number of switches is at $\sigma=0.065$, and there exists an interval $\sigma \in(0.05,0.08)$ in which the number of switches is more than 50. Moreover, the number of switches decreases with increasing intensity of noise from 0.08 to 0.14 . In Fig. 9(c), the number of switches are about 0 when the intensity of noise lies in the interval $(0.04,0.05)$. The maximum number of switches is about 150, and those over 100 lie in the interval $\sigma \in(0.065,0.12)$. When $r=2.1$, Fig. 9(e) shows that the number of switches increases with increasing intensity of noise from 0.07 to 0.09 and is 0 when $\sigma \in(0.04,0.07)$. However, the maximum number of switches is about 400 , and those exceeding 250 belong to $\sigma \in(0.1,0.135)$.

Next, we fixed the intensity of noise and varied the parameter $r$ from 2 to 2.55. Three different stable transient switches with $\sigma=0.04,0.08,0.1$ are shown in Figs. 9(b), 9(d) and 9(f), respectively. When $\sigma=0.04$, the switching times are 1 for $r \in(2,2.3)$. It means that the system switches from the initial oscillation to the attractor II, when the system finally stabilizes. The maximum number of switching times is about 20 at $r=2.32$ and 
switching times over 5 lie in the interval of $r \in(2.45$, 2.5). In particular, the switching times are 1 for a long interval $(r \in(2,2.3))$, which implies that the system has the attractor II (below DT) and the system has no locust outbreak. However, there exist two intervals whose switching times are 0 , the intervals are at $r \in(2.505,2.55)$, when the switching system does not switch. Moreover, the same phenomena occur when $\sigma=0.08,0.1$, when the switching times are 0 in different intervals [see Figs. 9(d) and $9(\mathrm{f})]$. Figures $9(\mathrm{~d})$ and $9(\mathrm{f})$ show that the maximum switching times are 180 and 250 respectively.

\section{Discussion and Biological Implications}

The modeling that we have described successfully captured essential features of polyphenic insects insofar as phase changes are related to population densities, intrinsic rates of increase, carrying capacities and predation. To achieve this we treated the insect populations as a Filippov system, variants of which have been widely used for modeling real world biological problems with switching surfaces [Tang et al., 2012; da Silveira Costa \& Faria, 2010; da Silveira Costa, 2007; da Silveira Costa \& Meza, 2006; Dercole et al., 2007]. The Filippov system is determined by different differential equations according to which the regions of phase space the solutions pass through [Filippov \& Arscott, 1988]. Similarly, based on the equilibria definitions of the Filippov system, discrete switching systems have been developed for pest control [Xiang et al., 2014a; Xiang et al., 2014b]. Based on an insect outbreak model which has bistability [Ludwig et al., 1978], we developed a discrete switching model to address desert locust outbreak problems and their complex dynamics.

By employing the equilibria analysis method introduced in [Tang et al., 2014], we first discussed the equilibria of the switching system according to six cases, which were $\left(S_{G_{2}}\right.$ bistability, $S_{G_{1}}$ refuge), ( $S_{G_{2}}$ bistability, $S_{G_{1}}$ bistability), $\left(S_{G_{2}}\right.$ bistability, $S_{G_{1}}$ outbreak), $\left(S_{G_{2}}\right.$ outbreak, $S_{G_{1}}$ refuge), $\left(S_{G_{2}}\right.$ outbreak, $S_{G_{1}}$ bistability), ( $S_{G_{2}}$ outbreak, $S_{G_{1}}$ outbreak). It is interesting that all cases of the discrete switching system (4) may exist within the same region, and that subsystem $S_{G_{2}}$ has a virtual equilibrium point. We can control subsystem $S_{G_{2}}$ such that the densities of the locust population fall below DT. Moreover, numerical investigations clarify that the pest population can be successfully controlled when a threshold control policy is applied (see Fig. 3).

When the two subsystems have outbreak equilibrium points (Case (vi)), the system has rich dynamics. The parameter bifurcation about $r$ shows that there are three kinds of attractors which can coexist at the same value of parameter $r$ [see Fig. 4(a)]. By extensive numerical bifurcation investigations, we found that when choosing different values for parameter $r$ and the initial values, attractors I-III have different regions as shown in Fig. 4(b). In particular, attractor III can switch to attractor II, finally, for some values of parameter $r$. In order to investigate the switching behavior for different values of parameter $r$ we selected to compute attractor switching points (see Figs. 5-7). The results showed that the switching behavior is sensitive to the parameter $r$ and initial values. For a better explanation of the factors influencing the numbers of attractor switches, we discussed these in relation to different values of the parameter $r$ and the intensity of noise $\sigma$ (see Figs. 8 and 9). The results showed that the number of attractor switches may be affected dramatically by small changes in the values of the intensity of noise, and in the values of $r$. Our results confirm that the switching frequencies depend heavily on the different noise intensities and different values of the intrinsic growth rate, which further indicates that the outbreak pattern of the locust populations may vary and could be very complex. This supports earlier suggestions that locust population dynamics could be chaotic [Cheke \& Holt, 1993, 1996].

\section{Acknowledgments}

This work was partially supported by the National Natural Science Foundation of China (11471201, S. Tang), and by the Natural Science Foundation of Hubei Province (2015CFB264, C. Xiang), and by the National Natural Science Foundation of China (61561019, C. Xiang), and by the National Natural Science Foundation of China (11601268, W. Qin).

\section{References}

Buhl, J., Sumpter, D. J., Couzin, I. D., Hale, J. J., Despland, E., Miller, E. \& Simpson, S. J. [2006] "From disorder to order in marching locusts," Science 312, 1402-1406. 
Cheke, R. A. [1978] "Theoretical rates of increase of gregarious and solitarious populations of the desert locust," Oecologia 35, 161-171.

Cheke, R. A. \& Holt, J. [1993] "Complex dynamics of desert locust plagues," Ecolog. Entomol. 18, 109-115.

Cheke, R. A. [1995] "Potential rates of increase of solitarious and gregarious phases of the African armyworm spodoptera exempta (lepidoptera: Noctuidae)," Ecolog. Entomol. 20, 319-325.

Cheke, R. \& Holt, J. [1996] "Chaos in desert locust plague dynamics," Populations and Patterns in Biology, pp. 42-45.

Cheke, R. A. \& Tratalos, J. A. [2007] "Migration, patchiness, and population processes illustrated by two migrant pests," Bioscience 57, 145-154.

Cheke, R. A., Tang, S. \& Tratalos, J. A. [2014] "Predator-prey population models of migrant insects with phase change," ICES J. Marine Science: Journal du Conseil 71, 2221-2230.

Cisse, S., Ghaout, S., Mazih, A., Ebbe, B., Ould, M. A., Benahi, A. S. \& Piou, C. [2013] "Effect of vegetation on density thresholds of adult desert locust gregarization from survey data in mauritania," Entomologia Experimentalis et Applicata 149, 159-165.

da Silveira Costa, M. I. \& Meza, M. E. M. [2006] "Application of a threshold policy in the management of multispecies fisheries and predator culling," Math. Med. Biol. 23, 63-75.

da Silveira Costa, M. I. [2007] "Harvesting induced fluctuations: Insights from a threshold management policy," Math. Biosci. 205, 77-82.

da Silveira Costa, M. I. \& Faria, L. D. B. [2010] "Integrated pest management: Theoretical insights from a threshold policy," Neotrop. Entomol. 39, 1-8.

Dercole, F., Gragnani, A. \& Rinaldi, S. [2007] "Bifurcation analysis of piecewise smooth ecological models," Theoret. Popul. Biol. 72, 197-213.

Dercole, F., Della Rossa, F., Colombo, A. \& Kuznetsov, Y. A. [2011] "Two degenerate boundary equilibrium bifurcations in planar Filippov systems," SIAM J. Appl. Dyn. Syst. 10, 1525-1553.

Drury, K. L. [2012] "Quantifying the effects of integrated pest management in terms of pest equilibrium resilience," Integrated Pest Management and Pest Control- Current and Future Tactics (INTECH Open Access Publisher), doi: 10.5772/30203.

Filippov, A. F. \& Arscott, F. M. [1988] Differential Equations with Discontinuous Righthand Sides: Control Systems, Vol. 18 (Springer Science \& Business Media).

Glendinning, P. [2015] "Classification of boundary equilibrium bifurcations in planar Filippov systems," MIMS EPrint 2015.50, Manchester Institute for Mathematical Sciences, University of Manchester, Manchester, UK.
Guttal, V. \& Jayaprakash, C. [2007] "Impact of noise on bistable ecological systems," Ecol. Model. 201, 420428.

Holt, J. \& Cheke, R. A. [1996] "Models of desert locust phase changes," Ecol. Model. 91, 131-137.

Kuznetsov, Y. A., Rinaldi, S. \& Gragnani, A. [2003] "One-parameter bifurcations in planar Filippov systems," Int. J. Bifurcation and Chaos 13, 2157-2188.

Ludwig, D., Jones, D. D. \& Holling, C. S. [1978] "Qualitative analysis of insect outbreak systems: The spruce budworm and forest," J. Anim. Ecol. 47, 315-332.

Moran, P. [1950] "Some remarks on animal population dynamics," Biometrics 6, 250-258.

Pedgley, D. (ed.) [1981] Desert Locust Forecasting Manual. Vol. I (Centre for Overseas Pest Research, London, HMSO).

Pedgley, D. E. (ed.) [1980] Desert Locust Forecasting Manual. Vol. II (Centre for Overseas Pest Research, London, HMSO).

Pener, M. [1991] "Locust phase polymorphism and its endocrine relations," Adv. Insect Physiol. 23, 1-79.

Pener, M. P. \& Simpson, S. J. [2009] "Locust phase polyphenism: An update," Adv. Insect Physiol. 36, $1-272$.

Ricker, W. E. [1954] "Stock and recruitment," J. Fisheries Board of Canada 11, 559-623.

Scheffer, M., Carpenter, S., Foley, J. A., Folke, C. \& Walker, B. [2001] "Catastrophic shifts in ecosystems," Nature 413, 591-596.

Serizawa, H., Amemiya, T. \& Itoh, K. [2009] "Noisetriggered regime shifts in a simple aquatic model," Ecol. Compl. 6, 375-382.

Simpson, S. J., McCaffery, A. R. \& Hägele, B. F. [1999] "A behavioural analysis of phase change in the desert locust," Biol. Rev. 74, 461-480.

Tang, S., Xiao, Y. \& Cheke, R. A. [2008] "Multiple attractors of host-parasitoid models with integrated pest management strategies: Eradication, persistence and outbreak," Theoret. Popul. Biol. 73, 181-197.

Tang, S., Liang, J., Xiao, Y. \& Cheke, R. A. [2012] "Sliding bifurcations of Filippov two stage pest control models with economic thresholds," SIAM J. Appl. Math. 72, 1061-1080.

Tang, S. \& Liang, J. [2013] "Global qualitative analysis of a non-smooth Gause predator-prey model with a refuge," Nonlin. Anal.: Th. Meth. Appl. 76, $165-180$.

Tang, S., Tang, G. \& Qin, W. [2014] "Codimension-1 sliding bifurcations of a Filippov pest growth model with threshold policy," Int. J. Bifurcation and Chaos 24, 1450122.

Tang, S., Pang, W., Cheke, R. A. \& Wu, J. [2015a] "Global dynamics of a state-dependent feedback control system," Adv. Diff. Eqs. 2015, 1-70. 
Tang, S., Tang, B., Wang, A. \& Xiao, Y. [2015b] "Holling II predator-prey impulsive semi-dynamic model with complex Poincaré map," Nonlin. Dyn. 81, 1575-1596.

Tratalos, J. A., Cheke, R. A., Healey, R. G., Stenseth, N. C. et al. [2010] "Desert locust populations, rainfall and climate change: Insights from phenomenological models using gridded monthly data," Clim. Res. 43, 229-239.

Uvarov, B. P. [1921] "A revision of the genus locusta, l. (= pachytylus, fieb.), with a new theory as to the periodicity and migrations of locusts," Bull. Entomol. Res. 12, 135-163.

Uvarov, B. et al. [1977] Grasshoppers and Locusts. A Handbook of General Acridology. Vol. 2. Behaviour, Ecology, Biogeography, Population Dynamics (Centre for Overseas Pest Research.).
Wang, X. \& Kang, L. [2014] "Molecular mechanisms of phase change in locusts," Ann. Rev. Entomol. 59, $225-244$.

Xiang, C., Xiang, Z., Tang, S. \& Wu, J. [2014a] "Discrete switching host-parasitoid models with integrated pest control," Int. J. Bifurcation and Chaos 24, 1450114.

Xiang, C., Xiang, Z. \& Yang, Y. [2014b] "Dynamic complexity of a switched host-parasitoid model with Beverton-Holt growth concerning integrated pest management," J. Appl. Math. 2014.

Xiao, Y., Miao, H., Tang, S. \& Wu, H. [2013] "Modeling antiretroviral drug responses for HIV-1 infected patients using differential equation models," $A d v$. Drug Deliv. Rev. 65, 940-953. 